\title{
REPORT OF THE TREASURER FOR THE PERIOD DECEMBER 1, 1937 TO NOVEMBER 30, 1938
}

\section{BALANCE SHEET}

November 30, 1938

\begin{tabular}{|c|c|c|}
\hline \multicolumn{3}{|l|}{ ASSETS } \\
\hline CASH IN BANKS: & \multirow{6}{*}{$\begin{array}{r}\$ 18,300.62 \\
124,318.99 \\
\end{array}$} & \multirow[b]{6}{*}{$\$ 142,619.61$} \\
\hline Corn Exchange Bank Trust Company . .. \$ 9,531.78 & & \\
\hline Bank for Savings $\ldots \ldots \ldots \ldots \ldots \ldots \ldots 2,782.29$ & & \\
\hline Union Dime Savings Bank ........... & & \\
\hline INVESTMENTS* $\ldots \ldots \ldots \ldots \ldots \ldots \ldots \ldots$ & & \\
\hline Total Assets $\ldots \ldots \ldots \ldots \ldots \ldots \ldots$ & & \\
\hline REserves and Surplus & & \\
\hline \multicolumn{3}{|l|}{ RESERVES : } \\
\hline Endowment Fund $\ldots \ldots \ldots \ldots \ldots \ldots \ldots \$ 70,481.07$ & & \\
\hline Bôcher Fund $\ldots \ldots \ldots \ldots \ldots \ldots \ldots \ldots, 2,182.46$ & & \\
\hline Cole Fund $\ldots \ldots \ldots \ldots \ldots \ldots \ldots \ldots \ldots, 3,323.82$ & & \\
\hline$\ldots \ldots \ldots \ldots \quad 4,319.16$ & & \\
\hline Reilly Fund ...... & & \\
\hline Life Memberships $\ldots \ldots \ldots \ldots \ldots \ldots \ldots, 5,763.88$ & & \\
\hline Sinking Fund $\ldots \ldots \ldots \ldots \ldots \ldots \ldots \ldots, 9,980.77$ & & \\
\hline Investment Losses $\ldots \ldots \ldots \ldots \ldots \ldots \ldots$ & & \\
\hline International Congress $\ldots \ldots \ldots \ldots \ldots \ldots$ & & \\
\hline Bulletin Reprinting and Index Fund .... & & \\
\hline Transactions Reprinting and Index Fund & & \\
\hline 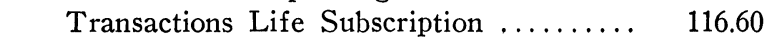 & & \\
\hline Colloquium $\ldots \ldots \ldots \ldots \ldots \ldots \ldots \ldots \ldots \quad 10,813.91$ & $\$ 129,983.67$ & \\
\hline SURPLUS AT NOVEMBER $30,1938 \ldots \ldots \ldots$ & $12,635.94$ & \\
\hline Total Reserves and Surplus ...... & & $\$ 142,619.61$ \\
\hline
\end{tabular}

Statement of General Receipts and General Disbursements GENERAL RECEIPTS :

Dues from Ordinary Memberships $\ldots \ldots \ldots \ldots \ldots \ldots . \$ 13,406.16$

Dues from Contributing Memberships ............ 1,124.96

Dues from Institutional Memberships ............ 6,455.00

Initiation Fees ............................ 629.86

Interest Income from Endowment Fund and General

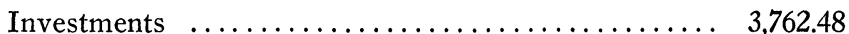

Miscellaneous .............................. 566.25

Total General Receipts ................... \$25,944.71

* Value of investments, exclusive of Brennan, Conlon, Sullivan and Davenport bonds and mortgages, based on market quotations on November 30,1938, is $\$ 88,666.80$. 
GENERAL DISBURSEMENTS :

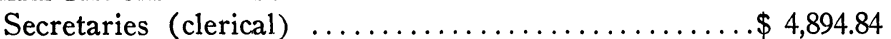

Treasurer .................................. 615.65

Officers' Traveling ........................... 476.40

Office Furniture and Fixtures .................. 169.22

Library ............................... 436.24

Membership Committee ...................... 28.93

Gibbs Lecture ......................... 111.48

American Journal Subvention $\ldots \ldots \ldots \ldots \ldots \ldots \ldots$. 2,500.00

Emergency $\ldots \ldots \ldots \ldots \ldots \ldots \ldots \ldots \ldots \ldots \ldots \ldots \ldots \ldots \ldots, \quad 61.60$

Total General Disbursements ............... 9,294.36

EXCESS OF GENERAL RECEIPTS OVER GENERAL DISBURSEMENTS $\$ 16,650.35$

SURPLUS AT DECEMBER 1, 1937

$\$ 18,142.71$

Additions :

Adjustment-Amortization of Investments ......\$ 70.79

Adjustment-Life Membership Reserve ........ 225.38

Adjustment-Transactions Subscription Reserve .. 1.19

Excess of General Receipts over General Disburse-

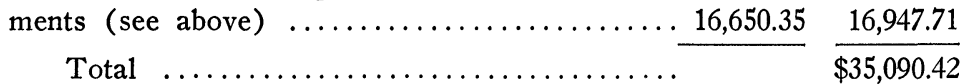

Deductions :

Appropriation to:

Investment Account for Bonds in Default ...... \$3,600.00

Reserve for Investment Losses .............. 665.44

Sinking Fund $\ldots \ldots \ldots \ldots \ldots \ldots \ldots \ldots \ldots \ldots, 4,000.00$

Bulletin Account .......................... 9,189.52

Transactions Account ................... 3,249.52

Transactions Reprinting Account ............ 500.00

Colloquium Account .................. 1,250.00

SURPLUS AT NOVEMBER $30,1938 \ldots \ldots \ldots \ldots \ldots \ldots \ldots \ldots . \ldots \ldots$

BuLletin

Receipts :

Subscription Sales .....................\$ 1,694.64

Reprint Sales ........................... $\quad 73.80$

Appropriation from General Receipts ........... $\quad 9,189.52$

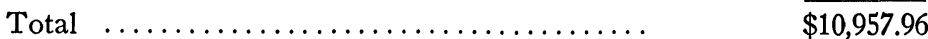

Disbursements :

Printing (2 numbers for 1937, 10 for 1938 ) $\ldots \ldots \ldots$ 6,757.79

List of Members ....................... 620.13

Salaries, Annuity and Pension ................ 3,093.49

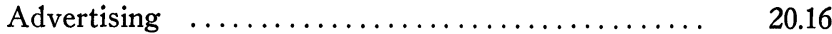

Editors' Traveling ....................... $\quad 5.00$

Miscellaneous-Office Furniture, Supplies, etc. ... $461.39 \quad 10,957.96$ 


\section{Bulletin Reprinting ANd Index}

BALANCE AT DECEMBER 1,1937 .

Sales of Back Volumes 389.60

BALANCE AT NOVEMBer 30, 1938

Transactions

Receipts :

Subscription Sales ....................... \$ 4,425.30

Back Volume Sales .......................... 684.30

Reprint Sales ......................... $\quad 208.22$

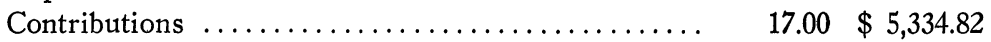

Appropriation from General Receipts ............ $3,249.52$

Total

$\overline{\$ 8,584.34}$

Disbursements :

Printing (1 number for 1937, 5 for 1938) . . . . . $\$ 6,499.92$

Salaries, Annuity and Pension ............ 1,755.45

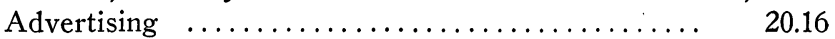

Miscellaneous-Postage, Supplies, etc. ........ 308.81

$8,584.34$

Transactions Reprinting and Index

BALANCE AT DECEMBER 1,1937

Appropriation from General Receipts

Total

$\$ 538.59$

Reprinting 318.16

BALANCE AT NOVEMBER 30, 1938

$\$ 220.43$

\section{Colloguium}

BALANCE AT DECEMBER 1, 1937

$\$ 9,806.32$

Receipts :

Sales of Colloquium Volumes ..............\$1,976.47

Sales of Colloquium Volume 16 (Bliss) for National

Research Council ......................... 133.39

Appropriation from General Receipts ........... 1,250.00

Total

$\frac{3,359.86}{\$ 13,166.18}$

Disbursements :

Printing (Volume 22) .................\$ 970.58

Salaries ............................. 969.49

Miscellaneous-Shipping, Supplies, etc. (including cost of shipping Volume 16 for National Research Council)

220.18

Circulars and Advertising .................. 62.13

Payment to National Research Council for Volume 16 (Bliss) ......................... 114.89

Colloquium Lecture Expenses .............. 15.00

BALANCE AT NOVEMBER $30,1938 \ldots \ldots \ldots \ldots \ldots \ldots \ldots \ldots \ldots$ 


\section{INTERNATIONAL CONGRESS}

Contributions

Disbursements

\section{Semicentennial}

BALANCE AT DECEMBER 1, 1937

Receipts :

Registration Fees, Ticket Sales, etc. ..........\$3,307.28

Publications ......................... 1,019.31

Appropriation from Sinking Fund

Total

Disbursements :

Meeting Expenses .................... \$3,650.57

Publications ........................ 4,295.25

Enlarged List of Members ................ 400.00

Special Funds

ENDOWMENT FUND

Total Principal Income

Balance at December 1, 1937 $\$ 70,456.07 \quad \$ 70,456.07$

Additions :

Income and Gifts

$2,444.39$

$25.00 \quad \$ 2,419.39$

Adjustment for Amortization of In-

vestments

45.52

Total

$\$ 72,945.98$

Deduction :

Interest Distributed to General Income 2,464.91

Balance at November 30, 1938 $\$ 70,481.07 \quad \$ 70,481.07$

$2,464.91$

BÔCHER FU.ND

Balance at December 1, 1937 $\$ 2,107.49 \$ 1,188.00$

$\$ 919.49$

Additions :

Income ......................

73.59

73.59 vestments 1.38

$\underline{\underline{\$ 1,188.00}} \stackrel{1.38}{\$ \$ 994.46}$

Balance at November 30, 1938 $\$ 2,182.46$

$\underline{\overline{11188.00}}$ 45.52 $\overline{\$ 70,481.07} \$ 2,464.91$ COLE FUND

Balance at December 1, 1937 \$3,211.35

$\$ 2,093.13$

$\$ 1,118.22$ Additions :

Income $\ldots \ldots \ldots \ldots \ldots \ldots \ldots \ldots . . . \ldots \ldots$. vestments

Balance at November 30, 1938 
MOORE FUND

Balance at December 1, 1937 $\$ 4,173.89$

$\$ 2,100.62 \$ 2,073.27$ Additions :

Income $\ldots . . . . . . . . . . . . . \ldots \ldots \ldots . . . . .142 .59$

142.59

Adjustment for Amortization of Investments $\ldots \ldots \ldots \ldots \ldots \ldots \ldots . . \ldots .62 .68$

Balance at November 30, $1938 \ldots \ldots \ldots \ldots . \overline{\$ 4,319.16} \overline{\$ 2,100.62} \overline{\$ 2,218.54}$ REILLY FUND

Balance at December 1, $1937 \ldots \ldots \ldots \ldots \ldots \ldots \$ 6,729.84 \$ 6,531.00 \$ 198.84$ Additions :

Income $\ldots \ldots \ldots \ldots \ldots \ldots \ldots \ldots \ldots$

Adjustment for Amortization of Investments ................

Received from Estate of Marion Reilly ................ 10,483.34 10,483.34

Balance at November 30, $1938 \ldots \ldots \ldots \ldots . \overline{\$ 17,733.35} \overline{\$ 17,014.34} \overline{\$ 719.01}$

Bennington P. Gill Treasurer

New York, N.Y.

510.56

November 30, 1938

\section{REPORT OF THE AUDITING COMMITTEE 1938}

We, the undersigned Auditing Committee, appointed by the American Mathematical Society, have this day audited the accounts of the Treasurer and in our opinion the following statement is correct:

CASH BALANCE AT DECEMBER 1, 1937

Corn Exchange Bank Trust Company ...........\$3,579.88

Bank for Savings ........................ 2,727.35

Union Dime Savings Bank .................. 5,868.30 \$12,175.53

Cash Receipts-December 1, 1937 to November 30, 1938-46,612.49

Interest on Savings Accounts in Bank for Savings and Union Dime Savings Bank ......................

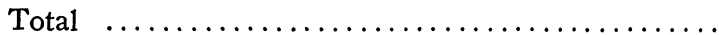

Cash Disbursements-December 1, 1937 to November 30, 1938

CASH BALANCE AT NOVEMBER 30, 1938:

Corn Exchange Bank Trust Company . . . . . . . . . . . . $\$ 9,531.78$

Bank for Savings . . . . . . . . . . . . . . . . . . . . . . 2,782.29

Union Dime Savings Bank ................... 5,986.55 